\title{
Different Bayesian Methods for Updating the Fatigue Crack Size Distribution in a Tubular Joint
}

\author{
H. Khalili ${ }^{a, b^{*} 1}$, S. Oterkus ${ }^{b}$, N. Barltrop ${ }^{b}$, U. Bharadwaj $^{c}$ \\ ${ }^{a}$ NSIRC, Cambridge, United Kingdom \\ ${ }^{b}$ University of Strathclyde, Glasgow, United Kingdom \\ ${ }^{c}$ TWI Ltd, Cambridge, United Kingdom
}

\begin{abstract}
Offshore platforms are prone to fatigue damage. To evaluate the fatigue damage, these platforms are periodically inspected during the in-service lifetime. Inspection activities provide additional information, which includes detection and measurement of crack size. A Bayesian framework can be used to update the probability distribution of the uncertain parameters such as crack size. After updating the distribution of the crack size, it is possible to improve the estimation of joint reliability. The main purpose of this study is to present different methods of Bayesian inference to update the probability distribution of the crack size using the inspection results and to demonstrate how the results are different. Two different methods are presented; analytical (conjugate) and numerical methods. The advantages and shortcomings of each method are discussed. To compare the results of the analytical and numerical methods, two different situations are considered; updating the crack size distribution for a particular joint and updating the crack size distribution for several joints that have almost the same conditions. Although the proposed methodology can be applied to different kinds of structures, an example of tubular joints in a specific jacket platform is presented to demonstrate the proposed approach and to compare the results of two methods.
\end{abstract}

Keywords: Jacket offshore platform, Fatigue crack size, Inspection results, Bayesian inference, Conjugate priors, Numerical method.

\footnotetext{
* Corresponding author:

E-mail address: hadi.khalili@strath.ac.uk
} 


\section{Introduction}

Jacket offshore platforms are one of the most common types of offshore structures that are utilized for oil and gas production in harsh environments. These platforms are usually constructed as truss frameworks in which tubular members are the structural elements. Fatigue damage in jacket platforms is most likely to occur at the welded tubular joints due to geometric discontinuity of the connections (which produces local tube wall bending and high-stress concentrations in these intersections) and the presence of small initial defects at the weld toe because of the welding process.

During the structure's lifetime, fatigue damage accumulates as the crack size increases. The accumulation of fatigue damage causes the probability of failure increases. To evaluate the state of fatigue damage, offshore platforms are periodically inspected. The information from inspection involves mainly the detection and measurement of crack size. After an inspection of a structure, the perception of the structure's condition can be improved. In general, the Bayesian framework is used to update the probability distributions of the fatigue uncertainties such as parameters of fracture mechanics and the fatigue crack size in joints. Using the updated distribution of the crack size, it is possible to update the estimation of the joint probability of failure.

Several studies have been performed to incorporate the inspection information to update the prior estimation of the fatigue uncertainties. Heredia and Montes (2004) developed a Bayesian framework for updating the probability distributions of the parameters of a fracture mechanics model and updating the crack size distribution in tubular joints by using the information from inspection reports. For this purpose, they defined an error model, which is a logarithmic difference between measured crack size during the inspection and crack size predicted by the fracture mechanics model. The error model was assumed to have a normal distribution with known mean and uncertain variance. In their model, a conjugate distribution for the error 
variance was considered. Based on these assumptions, they presented a closed-form expression based on a Bayesian approach to update the probability distribution of crack size in a tubular joint [1].

Karandikar et.al, (2012) performed a Bayesian inference using a random walk method to predict the remaining life of an aircraft fuselage panel subjected to repeated load cycles. They considered the Paris' law parameters, as uncertain along with the initial crack size. They generated a large number of random samples from the joint distribution of Paris' law parameters and initial crack size to produce the fatigue crack growth curve. The probability that each sample path was the true crack growth curve was assumed as the inverse of the number of random samples. They considered a uniform distribution for the prior estimation. Their likelihood function was represented as a non-normalized normal distribution to describe how likely it was to present the fatigue crack growth curve accurately. Having obtained the prior and likelihood, they calculated the posterior distribution using a numerical method for the fatigue crack growth curve [2].

Moan and Song, (2000) investigated the effect of inspection on updating the fatigue reliability of both inspected and uninspected joints in the offshore platforms. They also presented the effect of repeated inspections on fatigue reliability. They showed that even with a poor inspection technique, a rational success can be expected if the inspection can be repeated several times. In their study, the reliability of a single joint through crack detected and measured at a specific time was updated based on the numerical method [3].

Peng et.al, (2015) proposed a general framework for probabilistic prognosis and uncertainty management under fatigue cyclic loading. They considered several sources of uncertainties in the Bayesian updating framework. They conducted fatigue tests by using pre-installed piezoelectric sensors to obtain experimental data. They assumed prior distributions for initial 
crack size, stress intensity factor, and material property. They updated these distributions by using the laboratory test results [4].

In the above studies, the Bayesian updating has been performed through a numerical method and a likelihood function was defined to update the crack size distribution and the probability of failure. No comparison between the numerical and conjugate method has been carried out.

The main purpose of this study is to present and compare different Bayesian methods for updating the probability distribution of the crack size and to explain the relative advantages of each method. Therefore, the capability of the conjugate method can be investigated by comparing its results with the numerical method.

The Bayesian inference can be applied to any type of structure. However, the focus of this study is on tubular joints of the jacket platforms. The conjugate and numerical methods for updating the crack size distribution for a tubular joint are considered and the crack size distribution is updated when new information is available. Once the crack size distribution is updated, the probability of failure of the considered tubular joint is updated.

In practice, there are few inspection results available for each tubular joint due to the expensive cost of the inspection. Hence, a new methodology is presented to update the crack size distribution for different locations that have almost the same conditions. Therefore, the inspection results for each joint can be used not only for updating the distribution of a specific joint but also it can be used to update the distribution of crack size for joints which have similar conditions. A demonstration example is presented and the posterior distribution of the crack size is obtained by using both analytical and numerical methods.

It is noted that for redundant structures such as offshore jacket platforms, the reliability analysis at the system level is more applicable. However, this study focuses on the reliability estimation and updating at the component level by using Bayesian methods. 


\section{Fatigue Reliability Analysis}

In jacket platforms, fatigue cracks in tubular joints usually start at hot spot locations and gradually propagate through-thickness. For the safety of these structures, the fatigue damage of the tubular joints needs to be quantified. Due to the existence of several uncertainties in quantifying the fatigue damage process, a reliability approach is used. Reliability approaches are based on the calculation of failure probabilities. To calculate the failure probability, a limit state function needs to be defined based on the uncertain parameters involved in the fatigue process.

Two general approaches are widely used for fatigue analysis: S-N curve approach and fracture mechanics (FM). One of the significant shortcomings of the S-N method is that it cannot consider the changes in crack size during fatigue life. On the other hand, the FM approach relates the increase of crack size to the number of fatigue stress cycles and it is used to quantify the fatigue crack growth process [5]. Therefore, in this study, fatigue reliability analysis is performed based on the FM approach.

In the FM approach, the relationship between crack growth rate and stress intensity factor can be developed using the Paris law as [6],

$$
d a / d N=C \times(\Delta K)^{m}
$$

Where $a$ and $N$ represent the crack size and the number of load cycles, respectively. Moreover, $C$ and $m$ are the crack growth parameters, which depend on the material properties and loading conditions [7], [8]. In this equation, $\Delta K$ represents the stress intensity range which can be defined as [9],

$$
\Delta K=Y \times \Delta S \times \sqrt{\pi a}
$$

Where $\Delta S$ and $Y$ represent the stress range and the geometry function, respectively. In general, the geometry function depends on the crack geometry, structural geometry, and 
loading [8]. By plugging Eq. (2) into Eq. (1), the relation between crack size and the number of load cycles can be obtained as:

$$
\frac{d a}{(Y \sqrt{\pi a})^{m}}=C \times(\Delta S)^{m} \times d N
$$

By assuming $Y$ as a constant parameter [10] and by integrating Eq. (3) from the initial crack size $\left(a_{0}\right)$, to the crack size at time $t\left(a_{t}\right)$, the crack size value at time $t$ can be obtained as:

$$
a_{t}=\left\{a_{0}^{1-\frac{m}{2}}+\left(1-\frac{m}{2}\right) \times Y^{m} \times \pi^{\frac{m}{2}} \times C \times N \times \Delta S^{m}\right\}^{\frac{1}{1-\frac{m}{2}}}
$$

Due to the existence of several sea-state conditions, the platforms are exposed to several loading conditions. Therefore, the stress range in a platform is not constant and it varies for each sea state condition. Hence, the final crack size can be represented by using the expected stress range, as:

$$
a_{t}=\left\{a_{0}^{1-\frac{m}{2}}+\left(1-\frac{m}{2}\right) \times Y^{m} \times \pi^{\frac{m}{2}} \times C \times N \times E\left[\Delta S^{m}\right]\right\}^{\frac{1}{1-\frac{m}{2}}}
$$

After calculating the crack size based on the FM approach, the probability of failure can be calculated by using a limit state function. The limit state function represents the boundary between the safe and unsafe performance of a system or a component [11]. In this study, the crack size is treated as a failure criterion for the reliability calculations. It is assumed that failure occurs, as soon as the crack size is bigger than its critical value. Therefore, the fatigue limit state function is described as [10]:

$$
g=a_{c}-a_{t}
$$

Where $a_{c}$ represents the critical crack size. In this study, the critical crack size is taken as the wall thickness of the tubular joint [12]. The probability of failure is defined as the probability of fatigue limit state function is less than zero:

$$
P_{f}=P[g \leq 0]
$$


As can be seen from Eq. (6) and Eq. (7), the probability of failure is a function of the final crack size; $a_{t}$, which depends on the parameters such as initial crack size, crack growth parameters, geometry function, and the expected stress range (Eq. (5)). By plugging Eq. (5) into Eq. (6), the fatigue limit state function becomes:

$$
g=a_{c}-\left\{a_{0}^{1-\frac{m}{2}}+\left(1-\frac{m}{2}\right) \times Y^{m} \times \pi^{\frac{m}{2}} \times C \times N \times E\left[\Delta S^{m}\right]\right\}^{\frac{1}{1-\frac{m}{2}}}
$$

The reliability analysis depends on the choice of the uncertainties and their statistical distributions. Therefore, uncertainty modeling is very important for reliability calculations [11]. Several uncertainties exist in the fatigue life prediction of offshore structures such as:

- Estimation of environmental parameters (e.g. wave height, wave period)

- Calculation of hydrodynamic loads (e.g. Morison equation, drag and inertia coefficients)

- Estimation of structural response (e.g. structural mass, stiffness)

- Calculation of crack tip stress intensity factors (e.g. geometry function)

- Estimation of crack growth parameters (e.g. C and m)

- Estimation of the initial crack size

To consider the first three items which are related to general loading and response of the structure, global modeling of the structure is required [9]. One approach for considering these uncertainties is the Response Surface Method (RSM) [13]. However, in this study, only one single variable for the stress range is considered to model these uncertainties. The last three items which represent the uncertainties involved in the analysis of crack growth at the tubular joint are considered in detail.

The following uncertainties are considered in this paper:

1- Initial crack size: 
Initial crack size is an important variable that affects the fatigue life of a component. The initial crack size is not a well-known parameter and therefore there is uncertainty associated with the modeling of this parameter [14].

2- Crack growth parameter:

Fatigue tests indicate a considerable amount of scatter in the obtained fatigue capacities, which is as a result of material properties. There is always uncertainty in the definition of reasonable distributions for the material parameters based on available laboratory test results [14].

3- Geometry function:

Some empirical expressions for the geometry function are given by literature for simple welded joints. However, there is no analytical solution for the geometry function for complex geometries such as tubular joints and the experimental data shows a great deal of scatter for the geometry function [9]. To consider the uncertainty in the estimation of the geometry function, a stochastic variable $\left(\varepsilon_{Y}\right)$ is defined and the geometry function is multiplied by this random variable.

4- Stress range:

The stress range spectrum can be obtained by assuming relationships between wave height spectrum and wave stress spectrum. The sea wave loads during the platform service life are divided into a series of static sea states and each sea state can be described through the wave spectrum. For offshore jacket structures, the wave loading is considered narrow banded and the stress ranges are Rayleigh distributed.

To consider the uncertainties involved in the global analysis (e.g. environmental parameters, hydrodynamic loads, etc.), it is convenient to model the uncertainties with a single variable $\left(\varepsilon_{s}\right)$. The obtained stress range from the global analysis is then multiplied by this random variable [14]. 
Therefore, the limit state function can be written as:

$$
g=a_{c}-\left\{a_{0}^{1-\frac{m}{2}}+\left(1-\frac{m}{2}\right) \times\left(\varepsilon_{Y} Y\right)^{m} \times \pi^{\frac{m}{2}} \times C \times N \times \varepsilon_{S} E\left[\Delta S^{m}\right]\right\}^{\frac{1}{1-\frac{m}{2}}}
$$

Table 1 shows the distribution of the uncertain parameters considered in this study.

Table 1- Characteristics of the uncertain parameters [N, mm]

\begin{tabular}{|l|c|c|c|c|}
\hline \multicolumn{1}{|c|}{ Variable } & Type & Mean & COV $^{*}$ & Ref. \\
\hline Initial crack size, $a_{0}$ & Exponential & 0.11 & 1.0 & {$[14]$} \\
\hline Crack growth parameter, $C$ & Lognormal & $1.8 \times 10^{-12}$ & 0.63 & {$[15]$} \\
\hline $\begin{array}{l}\text { Stochastic parameter for the } \\
\text { geometry function, } \varepsilon_{Y}\end{array}$ & Lognormal & 1 & 0.1 & {$[15]$} \\
\hline $\begin{array}{l}\text { Stochastic parameter for the } \\
\text { stress range, } \varepsilon_{S}\end{array}$ & Lognormal & 1 & 0.1 & {$[11]$} \\
\hline
\end{tabular}

${ }^{*}$ Coefficient of Variation

Using Eq. (9) as the desirable limit state function and the introduced uncertainties in Table 1, the probability of failure can be obtained using Monte-Carlo simulation. The reliability can be calculated by using the probability of failure as:

$$
R=1-P_{f}
$$

\section{Bayesian Framework}

When additional information such as experimental data and inspection results become available, the obtained information can be used to improve the previous estimate of uncertain parameters. The framework for updating the distribution of estimated parameters is called the Bayesian framework [16]. A Bayesian framework is a powerful tool for uncertainty management. Bayesian inference provides a normative and formal method of belief updating when new information, becomes available [17].

The distributions that describe our knowledge before and after incorporating new data are called Prior and Posterior distributions, respectively. The posterior distribution of the uncertain parameter $(\theta)$, given new information $(x)$, can be obtained by using Bayes' theorem as [18]: 


$$
f(\theta \mid x)=\frac{f(x \mid \theta) \times f(\theta)}{f(x)}
$$

where:

- $f(\theta \mid x)$ : Posterior probability distribution, which is the probability of $\theta$ given $x$;

- $f(x \mid \theta)$ : Likelihood function which is the probability of observing $x$ (given $\theta$ );

- $f(\theta)$ : Prior probability distribution;

- $f(x)$ : Marginal probability of the new data. It is obtained by integrating out the uncertain parameter $(\theta)$ from the joint probability. Therefore, it is not dependent on the uncertain parameter, i.e.:

$$
f(x)=\int_{-\infty}^{\infty} f(x \mid \theta) \times f(\theta) \times d \theta
$$

$f(x)$ is considered as a normalization factor, i.e., a factor that makes the area under posterior distribution equal to one [18].

As can be seen from Eq. (11), in the Bayesian framework, the likelihood function and the prior distributions are the basis of the inference. In the Bayesian framework, the prior distribution represents our previous knowledge about the uncertain parameter before new information is available and the likelihood function, is defined to describe how likely is the new data to happen for a given uncertain parameter [19].

In general, numerical integration is required to obtain the normalization constant and therefore to obtain the posterior distribution. However, in some cases, the posterior distribution can be obtained analytically.

\subsection{Analytical (Conjugate) Method}

A prior distribution is said to be conjugate to a class of likelihood function if the resulting posterior distribution from Eq. (11) is in the same probability distribution family as the prior distribution [18]. Conjugate distributions are useful because the prior and posterior distributions have the same form so the contribution of the new data through the updating 
process can be easily quantified [18]. Since the posterior distribution is already known, the conjugate distributions provide tractable analytical results. Therefore, numerical integration to calculate Eq. (12) is not required. The use of conjugate priors allows obtaining the posterior distributions analytically. Commonly used conjugate distributions are shown in Table 2 [20].

Table 2- Common conjugate distributions

\begin{tabular}{|l|l|l|l|}
\hline \multicolumn{1}{|c|}{ Likelihood } & Uncertain parameter & \multicolumn{1}{|c|}{ Prior Distribution } & Posterior Distribution \\
\hline Binomial & $\pi$ & Beta & Beta \\
\hline Normal & $\mu\left(\sigma^{2}\right.$ known $)$ & Normal & Normal \\
\hline Normal & $\sigma^{2}(\mu$ known $)$ & Inverse Gamma & Inverse Gamma \\
\hline Normal & $\mu, \sigma^{2}$ & Normal Inverse Gamma & Normal Inverse Gamma \\
\hline Exponential & $\lambda$ & Gamma & Gamma \\
\hline
\end{tabular}

Prior distributions are classified as either informative or non-informative. The informative prior distribution is used when there is enough information about the uncertain parameter before collecting data. In reliability problems, informative prior distributions can be constructed using physical theory, computational analysis, and expert opinions (e.g. see [19] and [21]). In assessing probability distributions based on expert opinion, there are many potential biases that must be minimized [19].

Informative priors contain substantial information about the possible values of the model parameter. On the other hand, a non-informative prior distribution contains little information about the parameter of interest. When no expert opinion is available, it is recommended that a non-informative prior distribution be used [22]. It should be noted that this is rarely the case in practice, and the usage of non-informative priors in such cases can lead to conservative results [23]. However, it can be used when the analyst wants to use a prior that has little impact on the posterior. Non-informative priors, in general, are intended to let the data dominate the posterior distribution. The most common non-informative prior for a single parameter inference is the Jeffreys prior. The functional form Jeffreys prior depends on the likelihood function [23]. 


\subsection{Numerical Method}

The main shortcoming of the conjugate method is that in some cases, the uncertainty parameter does not have an associated conjugate prior. For example, generic databases often express uncertainty in terms of a lognormal distribution, which does not have any conjugate priors with likelihood function [23]. To use conjugate methods, the likelihood function and prior distribution must have specific standard distributions. In some cases, likelihood functions do not have known distributions. Therefore, using the conjugate method does not provide precise posterior distributions. Moreover, the conjugate approach contains assumptions that can influence the results. This influence may be predominant especially when there is sparse data that conflicts with the prior distributions [23].

On the contrary, the numerical method provides a more general approach for predicting posterior distributions. The normalization factor in Eq. (12) can be calculated numerically. In numerical methods, prior and posterior distributions do not have to have the same functional forms. When the prior distribution is not conjugate, the posterior distribution cannot be presented in an analytical form (closed-form). Therefore, the posterior distribution is not a standard distribution. Sampling from a non-standard distribution makes the updating procedure computationally more expensive.

\section{Case Study - Bayesian Updating of Crack Size Distribution for a Single Location}

The first step for updating of the crack size distribution is to select an appropriate prior. The prior crack size distribution can be assumed based on theoretical considerations, expert opinions, past experiences, or data reported in the literature [2].

In this study, a sampling method is used to obtain the prior distribution for crack sizes. Based on Eq. (5) the crack size at a specific time is a function of uncertain parameters such as initial crack size, crack growth parameter, geometry function, and the expected value of stress range. To obtain the prior distribution for crack size in the sampling method, a large number of 
samples are generated from the probability density function of each uncertain parameter as provided in Table 1. A large number of samples is used to include all relevant combinations of these uncertain parameters. For each set of random samples (e.g. for the $\mathrm{k}^{\text {th }}$ sample set: $a_{0 k}$, $C_{k}, \varepsilon_{Y k}$, and $\left.\varepsilon_{S k}\right)$, the crack size $\left(a_{t k}\right)$ is calculated based on Eq. (5). Here, the total number of simulations is chosen equal to $10^{5}$.

\subsection{Analytical (Conjugate) Method}

Figure 1 shows the histogram of simulated crack size distributions based on $10^{5}$ simulations after five years and the fitted exponential distribution. Since in the conjugate method, the prior distribution is restricted to the specific distributions, the fitted exponential distribution is used as an appropriate distribution for the crack size (before updating) with a probability density function as,

$$
f(a \mid \lambda)=\lambda e^{-\lambda a}
$$

Where $\lambda$ is the rate parameter and it is assumed as an uncertain parameter. The mean value $(\mu)$ of the exponential distribution is,

$$
\mu=\frac{1}{\lambda}
$$

As can be seen from Figure 1, the fitted exponential distribution overestimates the probability of smaller cracks, whereas it underestimates the probability of bigger cracks. 


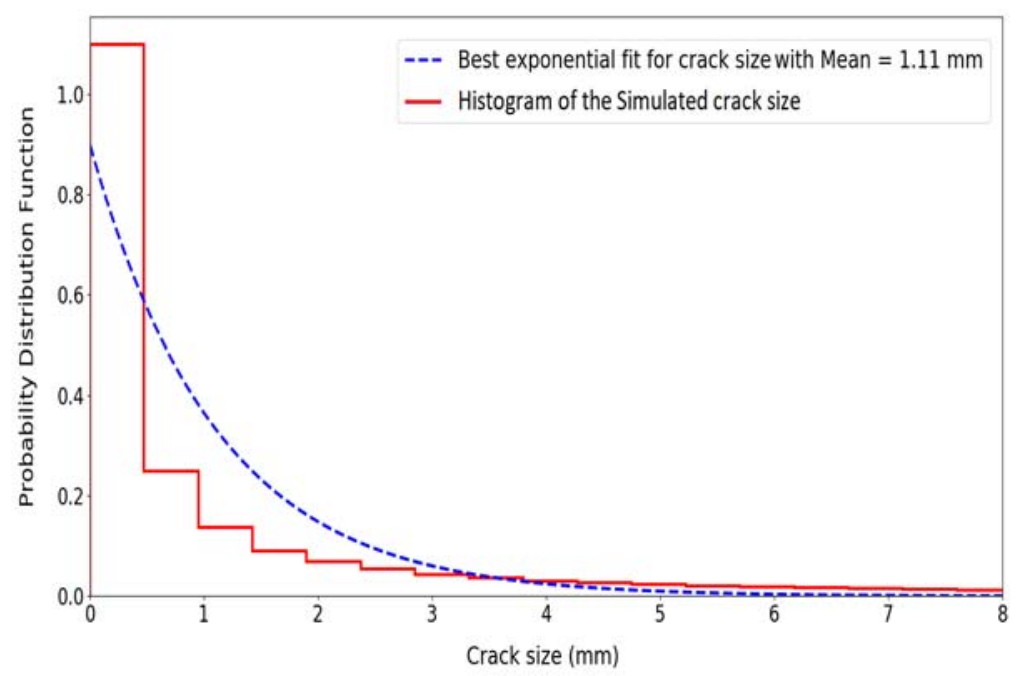

Figure 1- Histogram of the simulated cracks and fitted exponential distribution

It was mentioned that $\lambda$ is treated as an uncertain parameter which will be updated when new information is available. Based on Figure 1, one point estimation of $\lambda$ is obtained as $\lambda_{f i t}=\frac{1}{1.11}$. It is noted that the value of $\lambda_{\text {fit }}$ depends on the sample size. In this study, the uncertain parameter is the rate of exponential distribution $(\lambda)$, whereas the new information is the inspection results which contain the measurement of the crack size. Bayesian inference is employed to describe how the uncertainty in $\lambda$ changes from the prior distribution to the posterior distribution by incorporating the new information.

As can be seen from Table 2, for the exponential distribution with uncertain parameter $(\lambda)$, the conjugate prior and posterior distributions for $\lambda$ are Gamma distributions. The Gamma probability distribution function for $\lambda$ is presented as:

$$
\begin{gathered}
f(\lambda) \propto \operatorname{Gamma}(\alpha, \beta) \\
f(\lambda)=\frac{\beta^{\alpha}}{\Gamma(\alpha)} \lambda^{\alpha-1} e^{-\beta \lambda}
\end{gathered}
$$

Where $\alpha$ and $\beta$ are shape and rate parameters for the Gamma distribution, respectively. The expected value of the $\lambda$ can be obtained as [23]:

$$
E[\lambda]=\frac{\alpha}{\beta}
$$




\subsubsection{Prior Distribution}

In this study, Jeffreys non-informative prior will be used to describe the prior distribution. The Jeffreys non-informative prior for the exponential likelihood is a gamma distribution. In Jeffreys non-informative prior for the exponential distribution, both shape $\left(\alpha_{\text {prior }}\right)$ and rate parameters $\left(\beta_{\text {prior }}\right)$ are selected close to zero [23]. Moreover, these parameters are selected in a way that the expected value of $\lambda$ becomes equal to $\lambda_{\text {fit }}$, i.e.:

$$
\begin{gathered}
\alpha_{\text {prior }}=0.001, \quad \beta_{\text {prior }}=\alpha_{\text {prior }} \times 1 / \lambda_{\text {fit }} \\
E\left[\lambda_{\text {prior }}\right]=\frac{\alpha_{\text {prior }}}{\beta_{\text {prior }}}=\lambda_{\text {fit }}=\frac{1}{1.11}
\end{gathered}
$$

\subsubsection{New Information (Inspection Results)}

It is assumed that ten observations $\left(N_{\text {ins }}=10\right)$ of crack sizes $\left(a_{1}, a_{2}, \ldots, a_{10}\right)$ are available. A normal distribution with a mean value of $2 \mathrm{~mm}$ and a standard deviation of $0.2 \mathrm{~mm}$ is assigned to the inspection results. It is also assumed that these inspections are independent of each other.

\subsubsection{Posterior Distribution}

Having provided new information, the distribution of the uncertain parameter can be updated by using the Bayesian framework. According to the Bayes' theorem, the posterior is proportional to likelihood and prior:

$$
\begin{aligned}
f(\lambda \mid a) & \propto f(a \mid \lambda) \times f(\lambda) \\
f(\lambda \mid a) & \propto\left[\lambda e^{-\lambda a}\right] \times\left[\frac{\beta^{\alpha}}{\Gamma(\alpha)} \lambda^{\alpha-1} e^{-\beta \lambda}\right] \\
& \propto \lambda^{(\alpha+1)-1} e^{-(\beta+a) \lambda}
\end{aligned}
$$

Which is a gamma distribution with the following parameters:

$$
\begin{aligned}
f(\lambda \mid a) & \propto \operatorname{Gamma}\left(\alpha_{\text {prior }}+1, \beta_{\text {prior }}+a\right) \\
\alpha_{\text {posterior }} & =\alpha_{\text {prior }}+1, \quad \beta_{\text {posterior }}=\beta_{\text {prior }}+a
\end{aligned}
$$


The distribution provided in Eq. (20) is obtained for the posterior distribution for the uncertain parameter when only one new data is available. When several observations $\left(N_{\text {ins }}\right)$ of crack sizes are available, the parameters of gamma distribution can be obtained as [23]:

$$
\alpha_{\text {posterior }}=\alpha_{\text {prior }}+N_{\text {ins }}, \quad \beta_{\text {posterior }}=\beta_{\text {prior }}+\sum_{i=1}^{N_{\text {ins }}} a_{i}
$$

The updated expected value for $\lambda$ can be obtained by using Eq. (18) as:

$$
E\left[\lambda_{\text {posterior }}\right]=\frac{\alpha_{\text {posterior }}}{\beta_{\text {posterior }}}
$$

Figure 2 shows the prior and posterior distributions for the uncertain parameter $(\lambda)$. Here, a non-informative prior for the rate parameter is selected. In the non-informative prior, the rate parameter $(\lambda)$ can have any value between $(0, \infty)$. Therefore, the probability distribution approaches to zero. By incorporating the new information, the posterior distribution will be updated based on Eq. (21). Therefore, the posterior distribution becomes deviated from the initial prior distribution as can be seen from Figure 2.

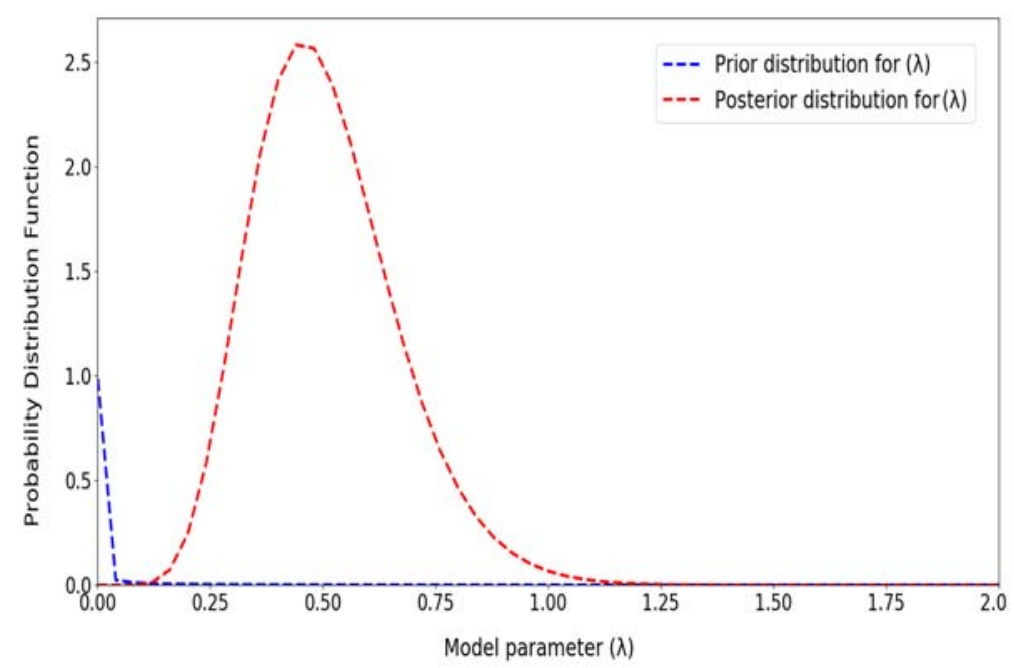

Figure 2- Prior and posterior distributions for the uncertain parameter

Based on Eq. (13), crack size distribution depends on the rate parameter. However, the rate parameter is an uncertain parameter which its distribution depends on $\alpha$ and $\beta$. This is schematically shown in Figure 3. 


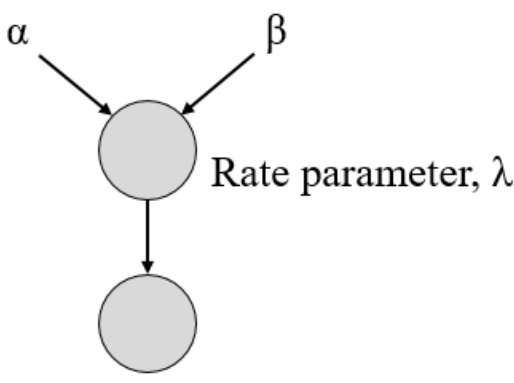

Crack size, a

Figure 3- Dependency of the crack size on the distribution of the rate parameter

After updating $\lambda$, the crack size distribution is updated based on predictive distribution. Predictive distribution for the crack size can be obtained as [23]:

$$
f(a)=\frac{\alpha_{\text {posterior }} \times \beta_{\text {posterior }}^{\alpha_{\text {posterior }}}}{\left(\beta_{\text {posterior }}+a\right)^{\alpha_{\text {posterior }}+1}}
$$

Figure 4 shows the crack size distributions before and after updating. As shown in the figure, the updated crack size distribution has a mean value of $1.97 \mathrm{~mm}$, which is close to the mean value of ten observations provided in Section 4.1.2.

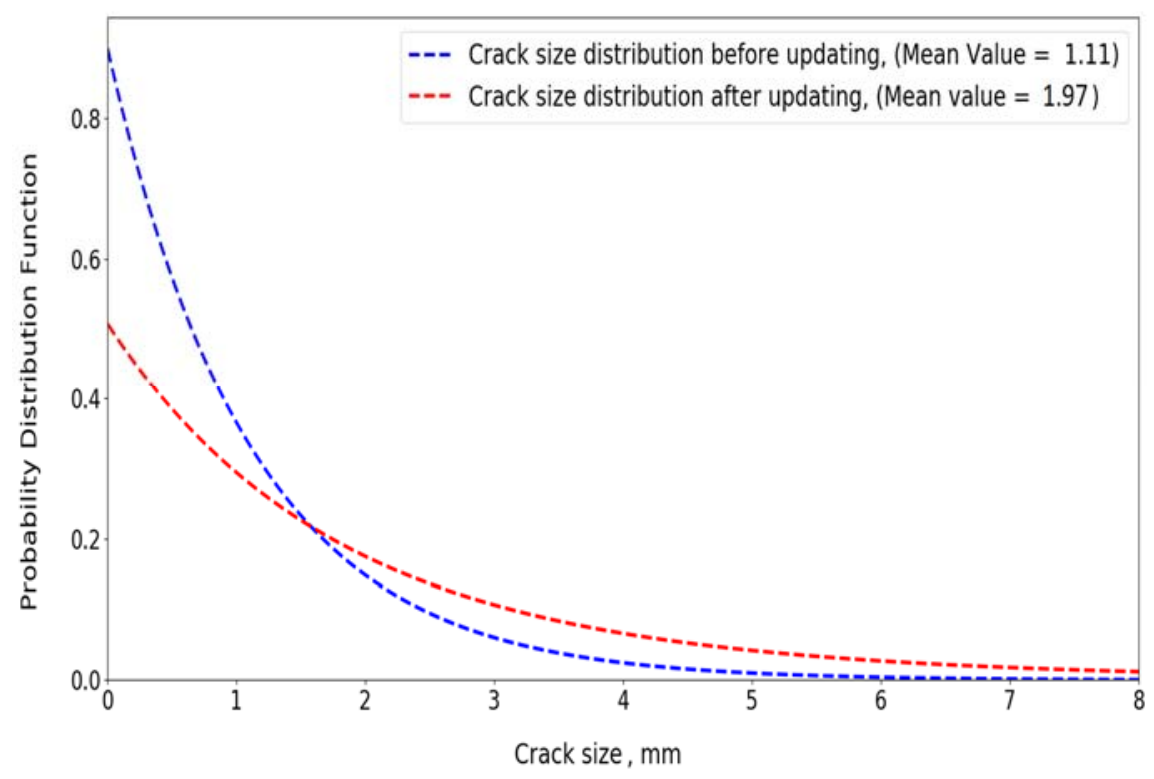

Figure 4- Crack size distributions before and after updating 


\subsection{Numerical Method}

In the numerical method, the posterior distribution is not a standard distribution and it cannot be presented in analytical form. Therefore, the normalization constant in Eq. (12) is calculated numerically. In this method, the posterior distribution is obtained by using Eq. (11).

\subsubsection{Prior Distribution}

As was mentioned a large number of crack sizes $\left(10^{5}\right)$ is predicted by using random samples of uncertain parameters. In the numerical method, instead of fitting any distribution, the histogram of the simulated crack size is used as a prior. Figure 5 shows the histogram of the simulated data which is used as a prior distribution for the crack size.

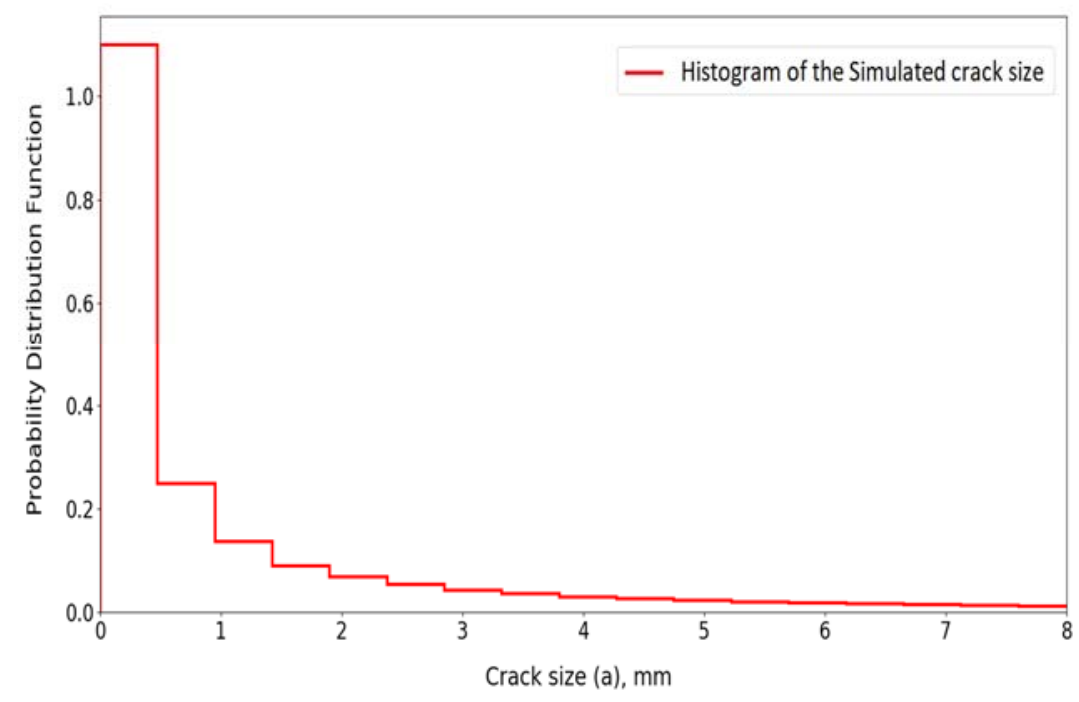

Figure 5- Prior crack size distribution for the numerical method

\subsubsection{Likelihood Function}

Due to the uncertainties in the fatigue phenomenon and crack size measurements, the crack size is not a certain parameter. The uncertainties involved in the fatigue process and also in the crack size measurements can be assumed based on expert's beliefs. Therefore, the likelihood function is defined based on the expert's belief to take into account the involved uncertainties. The likelihood function can be represented as a non-normalized normal distribution as [2]: 


$$
L=\exp \left(-\frac{\left(a-a_{m}\right)^{2}}{2 \sigma^{2}}\right)
$$

Where $a_{m}$ represents the measured crack size, $a$ is the crack size and $\sigma$ is the standard deviation of crack size (due to measurement and model uncertainty). For example, if the measured crack size is obtained equal to $1.8 \mathrm{~mm}$, the actual crack size probably is between 1.4 $\mathrm{mm}$ and $2.2 \mathrm{~mm}$. In this case, it is unlikely that the actual crack size is greater than $3 \mathrm{~mm}$.

The value of $\sigma$ in the likelihood function is estimated based on the expert's judgment about the uncertainty in the fatigue model and the accuracy of non-destructive testing (NDT). Since the value of $\sigma$ has a great effect on the likelihood function and posterior distribution, two different values for $\sigma$ are considered to see the effect of the expert's judgment on the posterior results. These cases are:

- Case (I): An accurate model for the predicted crack size and perfect measurements In this case, it is assumed that the model can estimate the real crack size with reasonable accuracy. Moreover, the measurement is performed with a high-quality tool. Therefore, a small standard deviation of $0.5 \mathrm{~mm}$ is considered for a likelihood function.

- Case (II): A less accurate model for the predicted crack size and less accurate measurement

In this case, it is assumed that the model can predict the real crack size with less accuracy. Moreover, the measured crack sizes are not very reliable. Therefore, a bigger standard deviation of $1 \mathrm{~mm}$ for the likelihood function is assumed.

Figure 6 shows the likelihood function for both cases. 


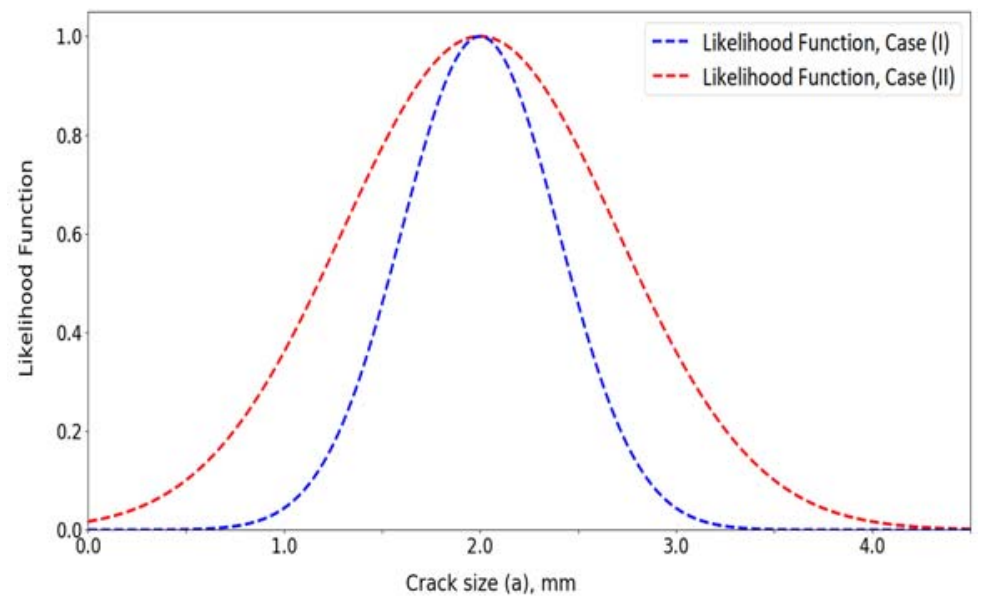

Figure 6- Likelihood functions for both accurate and inaccurate models

\subsubsection{Posterior Distribution}

According to Bayes' theorem, the posterior distribution is proportional to the prior distribution and likelihood function. It should be noted that, in the analytical method, the posterior distribution is updated only once by summing all the crack size information as shown in Eq. (21). However, in the numerical method, the posterior distribution needs to be updated for each observed crack size. Therefore, the probability distribution function of crack size is updated $N_{\text {ins }}$ times sequentially by using Eq. (11). The obtained posterior is assumed as the prior distribution for the next updating process. Therefore, the updating procedure for the numerical method is computationally more expensive than the analytical method.

To demonstrate how the crack size distribution is updated, the same observations in Section 4.1.2 are considered. Figure 7 and Figure 8 show the posterior crack size distributions after the updating process for both likelihood cases described in Section 4.2.2. 

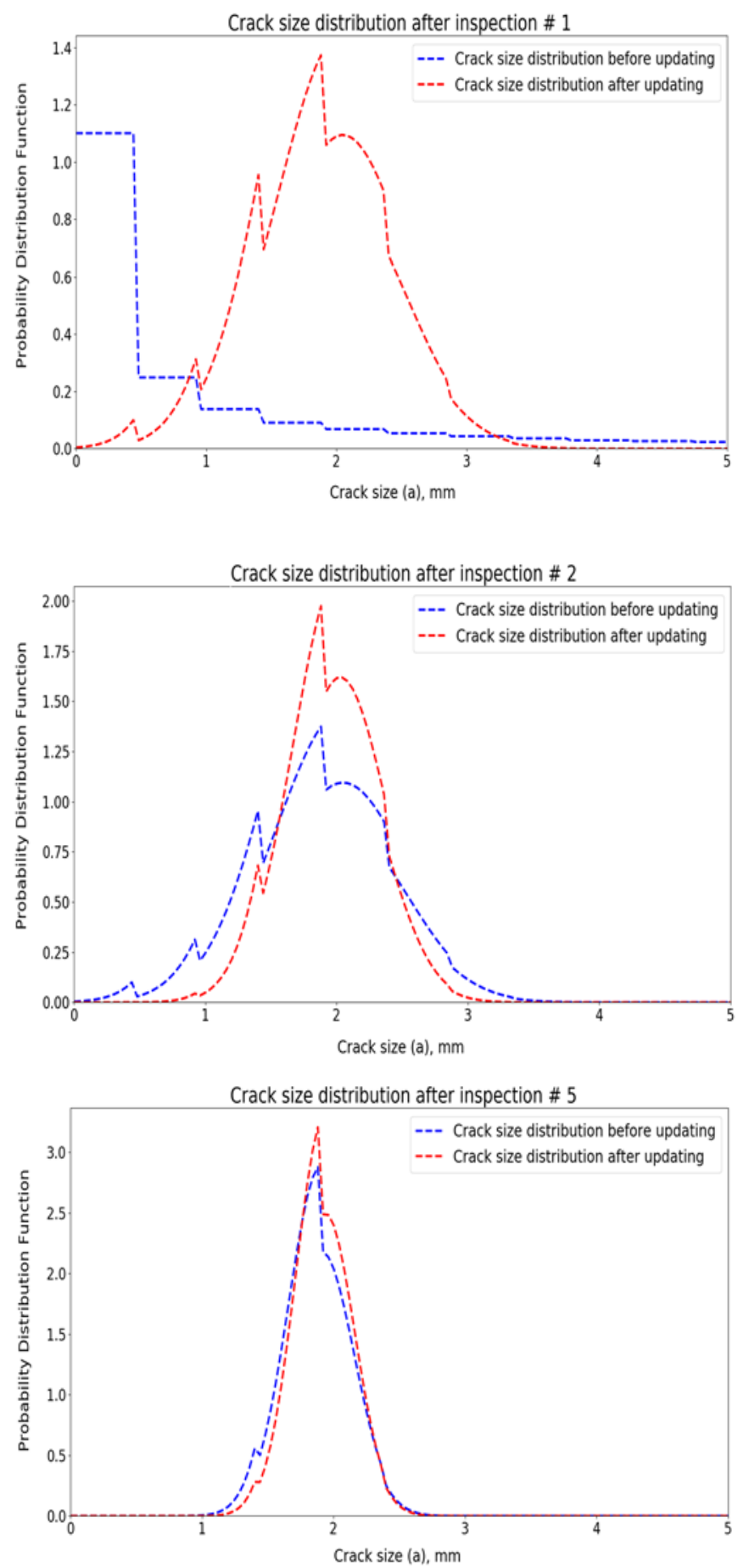


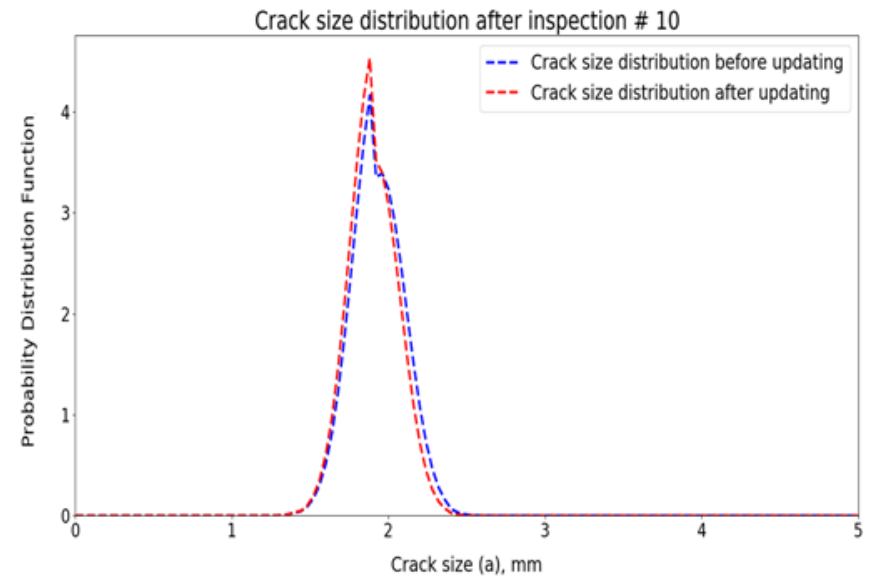

Figure 7- Crack size distributions after several inspections for likelihood function-Case (I)
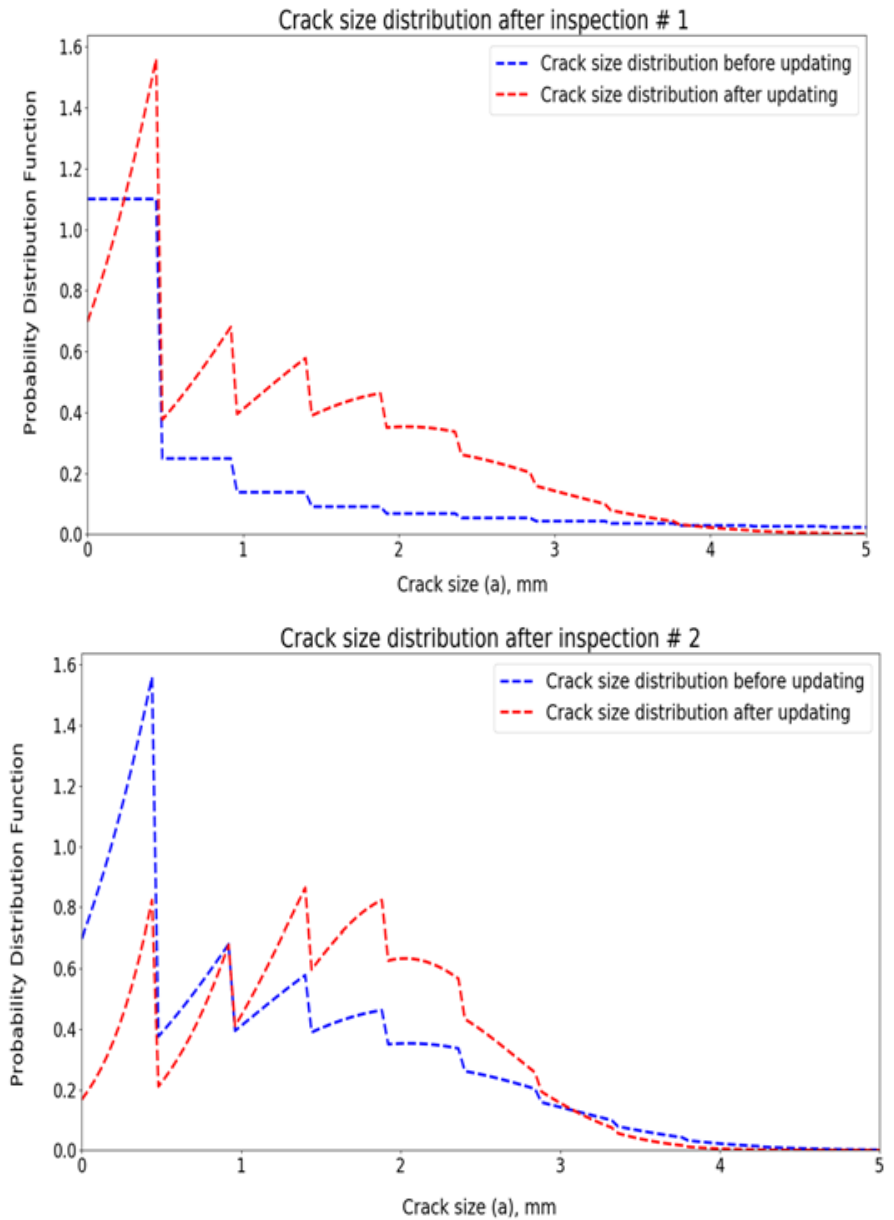

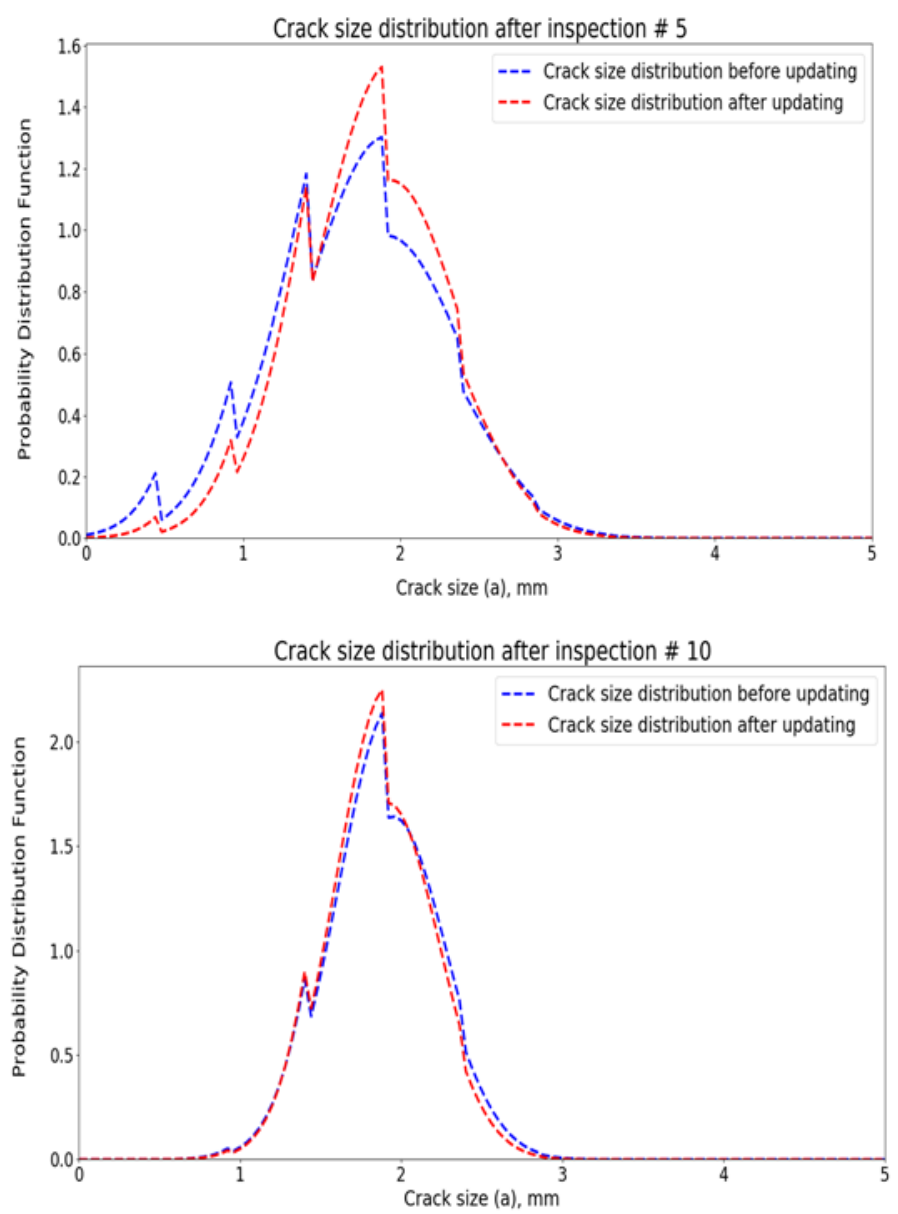

Figure 8- Crack size distributions after several inspections for likelihood function-Case (II)

Figure 7 and Figure 8 illustrate that the posterior distributions are shifted towards the observations after almost five inspections, i.e. the observed data dominates the posterior distributions. Additionally, it is also observed that the expected value for the crack size is around $2.0 \mathrm{~mm}$ in both figures, which is close to the mean of the observed crack sizes. Therefore, if enough data is available, the posterior distribution is not sensitive to the prior selection. However, due to the high cost of underwater inspections, there may not be several inspections available for a specific joint. Most of the time, there is only one or two inspection results for each joint. As represented in Figure 7 and Figure 8, for the first and second updates, prior distribution has a great impact on the posterior distribution. Therefore, the prior distribution should be selected based on reasonable assumptions. 


\subsection{Comparing the Results}

Figure 9 shows the posterior distributions of crack sizes for both conjugate and numerical methods. Although the obtained mean values for the methods are not substantially different $\left(\mu_{\text {Conjugate }}=1.97, \mu_{\text {Numerical }}=1.88 \mathrm{~mm}\right)$, the shape of the crack size distribution is different.

The reason is that the posterior distribution is restricted to a specific distribution shape in the conjugate method. This is an important disadvantage of using the conjugate methods since the prior distributions have a great impact on the posterior distributions. Moreover, in the conjugate method, the simulated crack size is approximated with a fitted exponential distribution which is overestimating the probability of smaller cracks while underestimating the probability of bigger cracks. Therefore, when the prior distribution is not precise, the posterior distribution may not be accurate. It is important to note that conjugate priors involve making relatively strong assumptions. Indeed, conjugate priors minimize the impact of the data on the posterior. However, with a small amount of data, that is insufficient to define the distribution, the analytical method with its assumed posterior distribution shape can be employed.

It is also observed that the posterior distribution shapes provided in Figure 9.b are very similar to the likelihood functions given in Figure 6. In fact, in the numerical method, the observed data dominates the posterior distributions. The effect of likelihood function on the posterior distribution can also be understood from Figure 9.b. The likelihood function for Case (II) which has a bigger uncertainty widens the posterior distribution. Using a larger uncertainty results in a bigger probability of failure. 


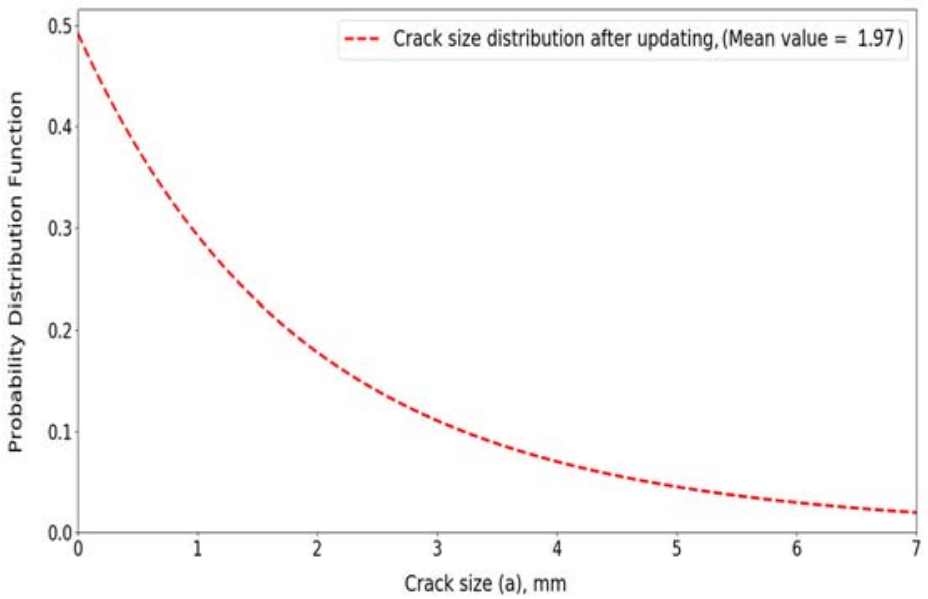

(a)

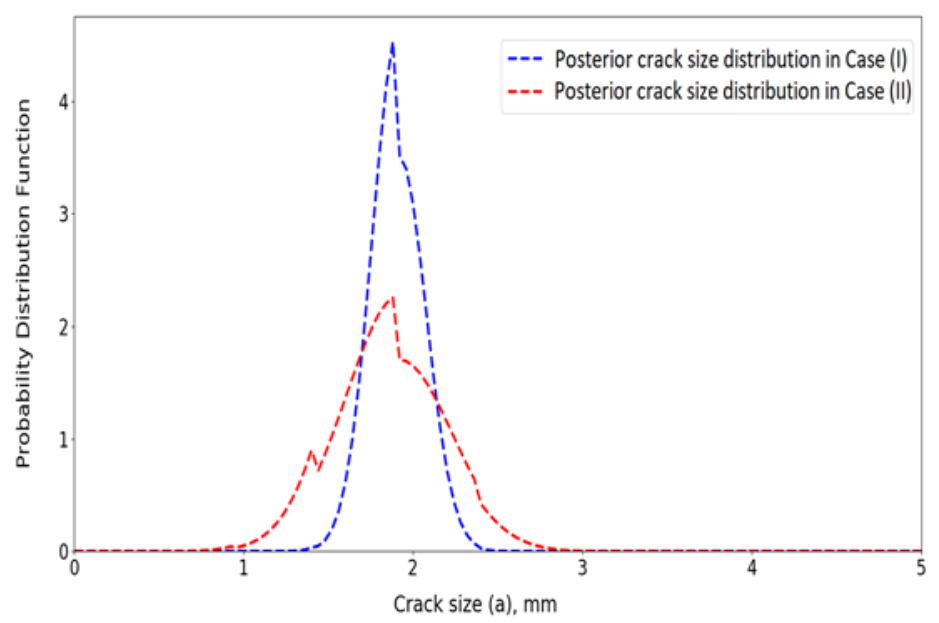

(b)

Figure 9- Posterior crack size distributions, (a) Conjugate approach, (b) Numerical approach

In the numerical method, the observed data dominates the posterior distribution shape, whereas, in conjugate methods, the prior distribution has a great effect on the posterior distribution and the effect of observed data is minimized. Since the numerical method allows us to incorporate the effect of the new data (inspection results) on the posterior distribution, numerical methods are preferred when sufficient data is available. However, the numerical method is computationally more expensive when several inspection results are available. 


\subsection{Updating the Reliability as a Function of Time}

As mentioned earlier, once the crack size distribution is updated, the probability of failure (reliability) of the considered tubular joint can be updated. After updating the crack size distribution, the component probability of failure can be updated by using the fatigue limit state and Monte Carlo simulations. Failure happens when the crack size reaches a critical size $\left(a_{c}\right)$, which is assumed equal to the thickness of the joint.

Consider $a$ as the crack size at time $t$ and $f(a)$ as the corresponding probability distribution. If $f_{0}(a)$ denotes the crack size distribution before updating and $f_{1}(a)$ indicates the distribution after updating, the probability of failure before updating is estimated by:

$$
P_{f_{0}}=P\left[a \geq a_{c}\right]=1-\int_{0}^{a_{c}} f_{0}(a) \times d a
$$

The probability of failure after updating is estimated by:

$$
P_{f_{1}}=P\left[a \geq a_{c}\right]=1-\int_{0}^{a_{c}} f_{1}(a) \times d a
$$

\section{Case Study - Bayesian Updating of Crack Size Distribution for Multiple Locations}

In real situations, there are few inspection results available for each tubular joint due to the expensive cost of the inspection. The purpose of this section is to update the crack size distribution for different locations that have almost the same conditions. It is assumed that these joints have identical configurations with the same material properties and they are subjected to almost the same stress range. When the inspection results for these similar joints are available, the posterior distribution for this group of joints can be obtained by using conjugate or numerical methods.

\subsection{Prior Distribution}

To assign a prior distribution for the crack size, a sampling method as explained in Section 4 is used. Distributions of the uncertain parameters are presented in Table 1. However, since 
the stress range is not the same at different locations, a bigger coefficient of variation is assumed for stress range as $\varepsilon_{S}=0.25$.

The histogram of the simulated crack sizes and the fitted exponential distribution are assumed as the prior distribution for numerical and conjugate methods, respectively. The prior distributions shown in Figure 10 are obtained based on $10^{5}$ simulations.

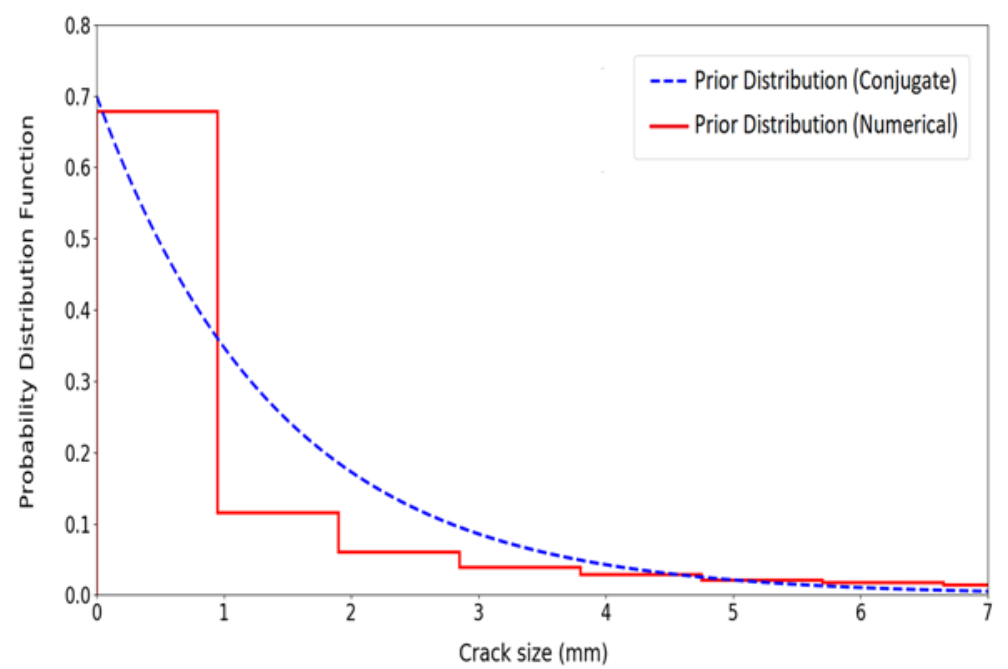

Figure 10- Prior crack size distributions for multiple locations in conjugate and numerical methods

\subsection{New Information (Inspection Results)}

It is assumed that there are twenty tubular joints with similar conditions. To demonstrate the methodology, it is assumed that five independent inspection results are available for each tubular joint. Therefore, in total 100 inspection results $\left(N_{\text {ins }}=100\right)$ are available. A normal distribution with a coefficient of variation of 0.2 is assumed for these five inspection results. It is also assumed that the mean value of each tubular joint is different.

\subsection{Posterior Distribution}

\subsubsection{Conjugate Method}

Prior and posterior distributions for the uncertain parameter $(\lambda)$ are represented by gamma distributions. After updating the distribution of $\lambda$, the crack size distribution is obtained by 
using Eq. (23). Figure 11 shows the crack size distributions before and after incorporating the inspection results.

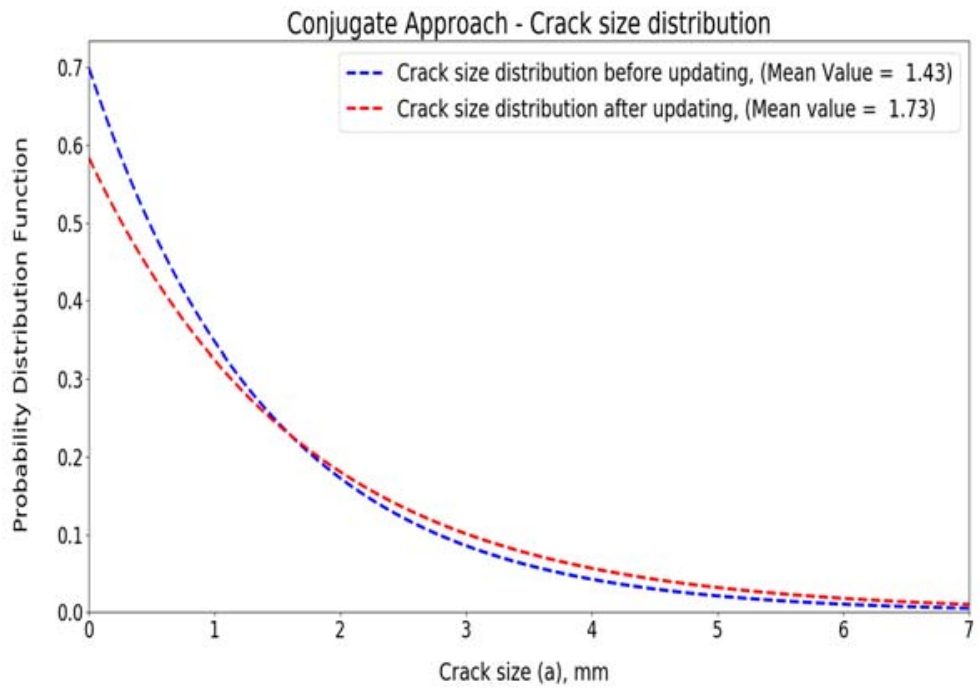

Figure 11- Crack size distributions for multiple locations in the conjugate method

\subsubsection{Numerical Method}

As it was mentioned earlier in Section 4.2, the likelihood function is defined by Eq. (24). The standard deviation of crack size is assumed as $\sigma=0.8 \mathrm{~mm}$. Based on Bayes' theorem, the posterior distribution is obtained by using the prior distribution and likelihood function as presented in Eq. (11). Moreover, as shown in Figure 9.b, the posterior distribution shape in the numerical method is similar to the likelihood function presented in Figure 6. It was assumed that the inspection results are available for twenty tubular joints. Figure 12 shows the crack size distributions for some joints before and after incorporating the inspection results. 


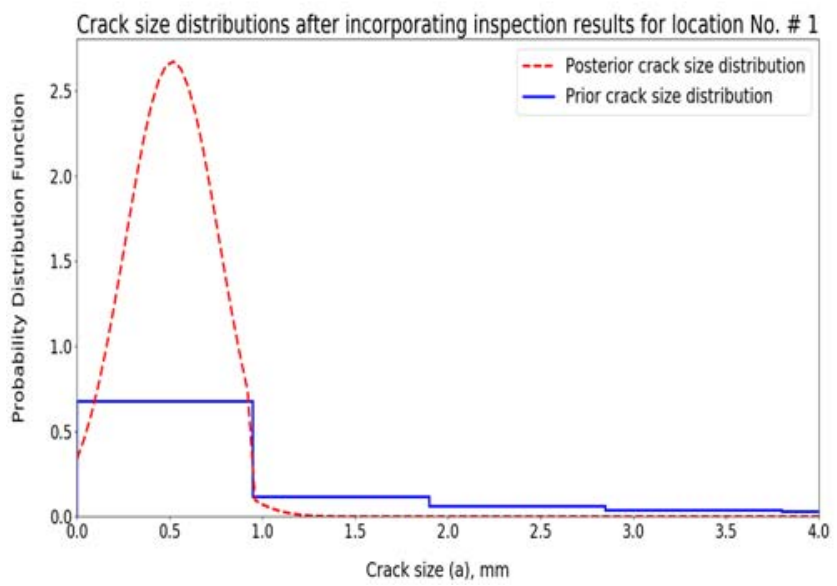

(a)

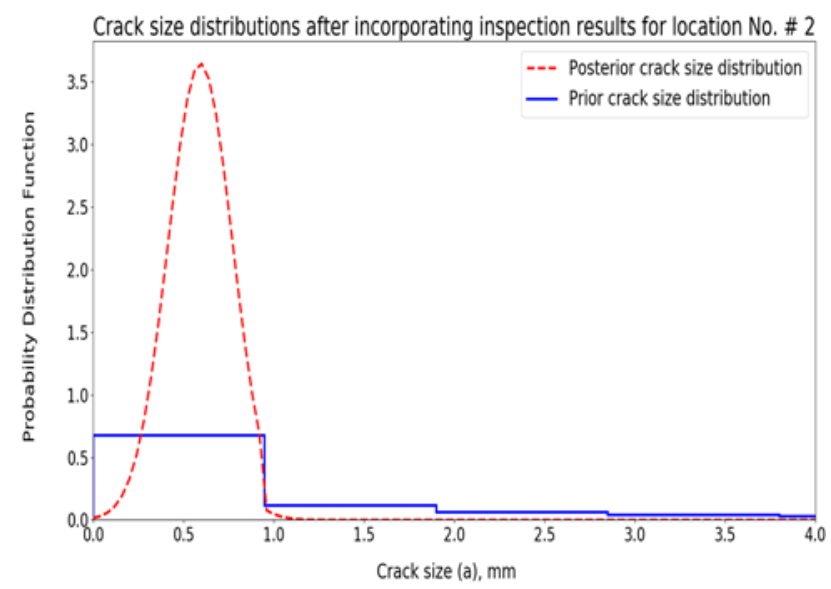

(b)

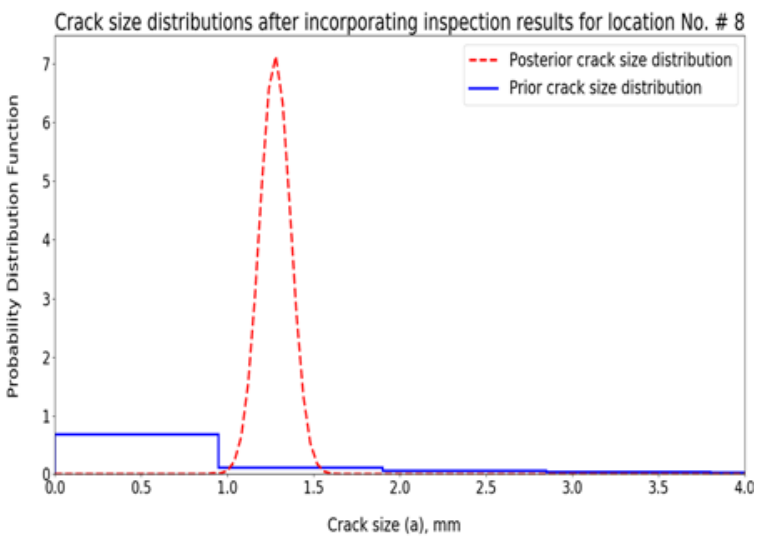

(c) 


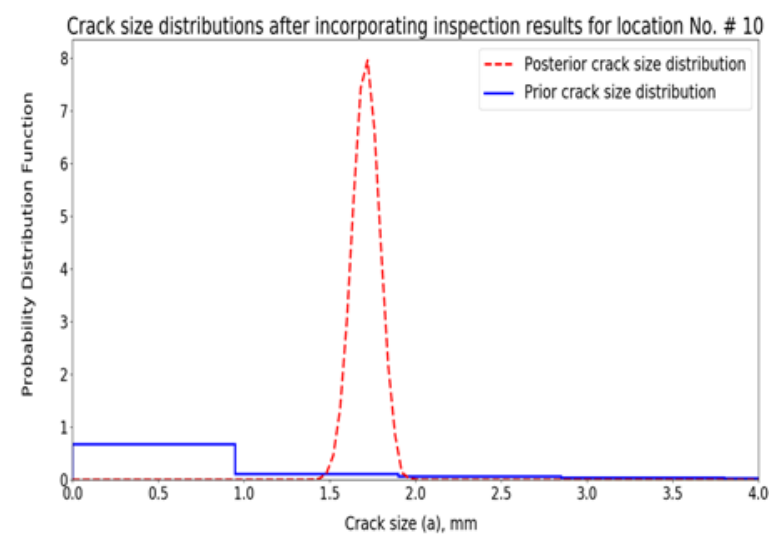

(d)

Figure 12- Crack size distributions for different locations, (a) joint \#1, (b) joint \#2, (c) joint \#8 and (d) joint \#10

In Figure 12 the blue line is the prior distribution for all similar joints in the platform and it is assumed similar for all the joints. However, for each joint, the specific inspection data for that joint is used. Therefore, the posterior distribution for each joint is different as presented by red dashed lines.

By combining all posterior distributions for each location and normalizing the area below the combined distribution, a posterior distribution for all locations can be obtained. Figure 13 illustrates the prior and posterior crack size distributions for all tubular joints.

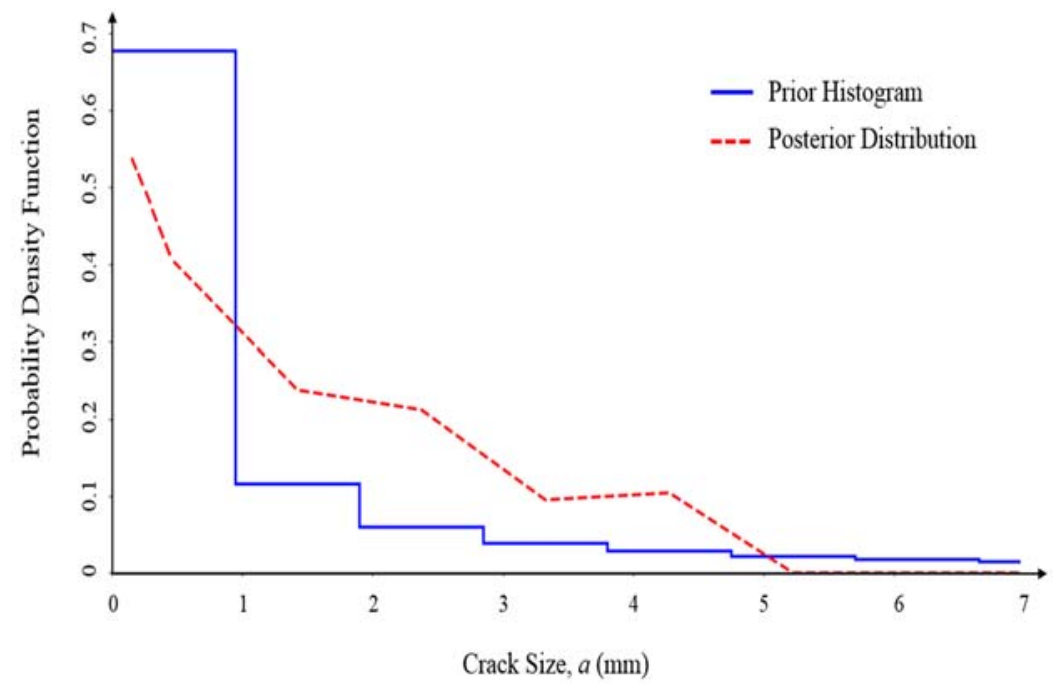

Figure 13- Crack size distributions for multiple locations in the numerical method 


\subsection{Comparing the Results}

Figure 14 shows the posterior crack size distributions for a group of twenty similar joints based on the conjugate and numerical methods. It is observed that, if the results of several locations are combined, the posterior distribution in the numerical method approaches to the conjugate posterior distribution. Note that the posterior for each location is similar to normal distribution. It is also found that both numerical and conjugate methods result in similar distribution. Therefore, posterior distribution in the conjugate method might be preferred since it is much easier and less time-consuming for multiple locations.

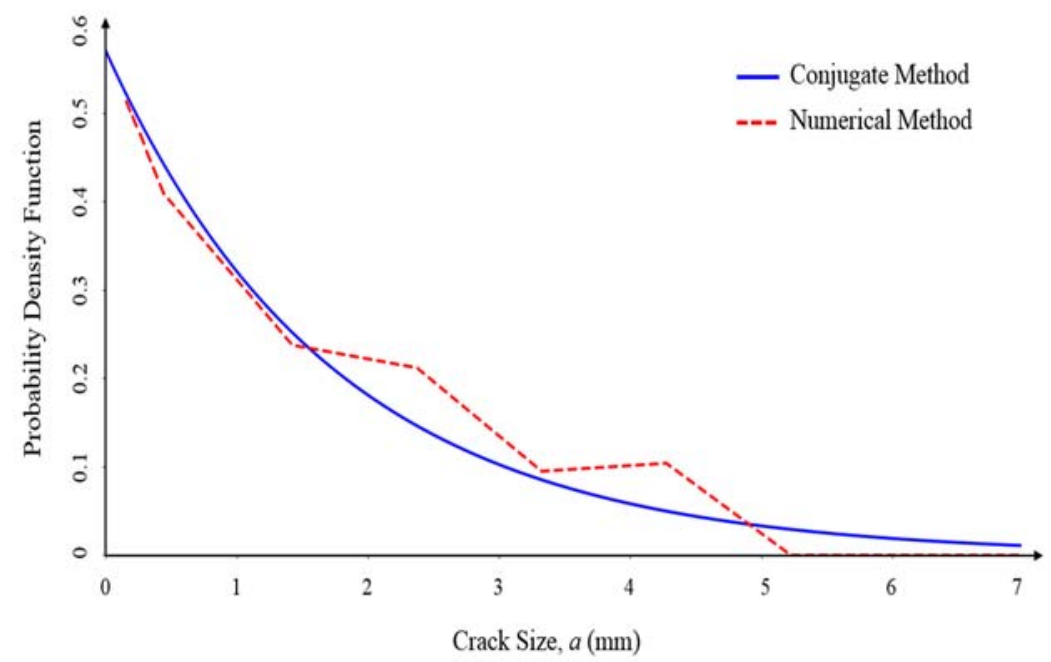

Figure 14- Posterior crack size distributions for multiple locations in both methods

\section{Conclusion}

In this study, two different methods for updating the crack size distribution are introduced; analytical method and numerical method. The main advantage of using the conjugate method is having known posterior distributions. The posterior can be easily obtained when new information is available. However, conjugate priors contain substantial assumptions. In fact, in the conjugate method, priors have a strong influence on the posterior compared to the influence of the data. Conjugate methods could be useful for cases where the observations are insufficient to estimate the distribution shapes. 
A numerical method is a general approach for Bayesian updating which is used to obtain the posterior by multiplying likelihood function and prior distribution directly. By using the numerical method, data dominates the posterior distribution. Hence, the numerical method is preferred when sufficient data is available. However, the numerical method is computationally expensive.

Since, in reality, there are only one or two inspections for each joint, a new method is presented to combine the inspection results of the similar joints to update the distribution of these similar joints. In fact, by using this method, the inspection results can be used to update other joint distribution and it tries to maximize the benefit of each inspection. When the inspection data is available for several joints, the results of the numerical method approach those of the conjugate method. Since the posterior distribution in the conjugate method can be obtained easier than the numerical method, the conjugate method might be preferred in this case.

\section{Acknowledgements}

This publication was made possible by the sponsorship and support of Lloyd's Register Foundation. The Foundation helps to protect life and property by supporting engineeringrelated education, public engagement, and the application of research. The work was enabled through, and undertaken at, the National Structural Integrity Research Centre (NSIRC), a postgraduate engineering facility for industry-led research into structural integrity established and managed by TWI through a network of both national and international Universities. 


\section{References}

[1] Heredia-Zavoni E.; Montes-Iturrizaga R., "A Bayesian Model for the Probability Distribution of Fatigue," Journal of Offshore Mechanics and Arctic Engineering, p. Vol. 126, 2004.

[2] Karandikar J.M., Kim N. H., Schmitz T.L., "Prediction of remaining useful life for fatigue-damaged structures using Bayesian inference," Engineering Fracture Mechanics Journal, p. 588-605, 2012.

[3] Moan T., Song R., "Implications of Inspection Updating on System Fatigue Reliability of Offshore Structures," Journal of Offshore Mechanics and Arctic Engineering, p. Vol. 122, 2000.

[4] Peng T., He J., Xiang Y., Liu Y., Saxena A., Celaya J., Goebel K., "Probabilistic fatigue damage prognosis of lap joint using Bayesian updating," Journal of Intelligent Material Systems and Structures, pp. Vol. 26, p.965-979, 2015.

[5] Almar-Naess, "Fatigue handbook for offshore steel", Tapir publication, 1985.

[6] Paris P.C, Erdogan F., "A critical analysis of crack propagation laws," Basic Engineering Journal, ASME, 1960.

[7] Rajasankar J., Iyer N.R., Appa Rao T.V.S.R., "Structural integrity assessment of offshore tubular joints based on reliability analysis," International Journal of Fatigue, pp. p.609-619, 2003.

[8] Ahmadi H., Lotfollahi-Yaghin M., Aminfar M.H., "Effect of stress concentration factors on the structural integrity assessment of multi-planar offshore tubular DKT-joints based on the fracture mechanics fatigue reliability approach," Ocean Engineering, p. p.1883-1893, 2011.

[9] Aghakouchak A.A., Stiemer S.F., "Fatigue reliability assessment of tubular joints of existing offshore structures," Canadian Journal of civil engineering, p. Vol. 28, 2001.

[10] Lin H., Chen G., Wang Z., Yang L., "Integrality Assessment of Tubular Joints in Aging Offshore Platform Based on Reliability Theory," Advanced Materials Research, pp. pp 238-242, 2012.

[11] Khalili H., Oterkus S., Barltrop N., Bharadwaj U., Tipping M., "System reliability calculation of jacket platforms including fatigue and extreme wave loading," Trends in the Analysis and Design of Marine Structures, Parunov \& Guedes Soares $\mathbb{C}$ Taylor, 2019.

[12] Wang W., Shi Y., Wang C., Li S., "A new method for system fatigue reliability analysis of offshore steel jacket," Advances in Structural Engineering, p. Vol. 9, 2006.

[13] Mosayyebi A.R., Aghakuchak A.A., "Fatigue reliability analysis of tubular joints of offshore structures, using response surface method," Asian journal of civil engineering (building and housing), vol. 1, no. 1, pp. 75-87, 2000.

[14] "Guideline for structural reliability analysis: application to jacket platforms," DNV report No. 953203, 1995.

[15] Siddiqui N.A., Ahmad S., "Fatigue and fracture reliability of TLP tethers under random loading," Marine Structures, pp. p.331-352, 2001.

[16] Melchers, Robert E., "Structural reliability analysis and prediction", Wiley, 1999.

[17] Gelman A., Carlin J.B., Stern H.S., Rubin D.B., "Bayesian data analysis", 2nd edition, Chapman and Hall/CRC Press, 2009.

[18] K.-V. Yuen, "Bayesian Methods for Structural Dynamics", John Wiley \& Sons, 2010.

[19] Hamada M.S., Wilson A.G., Reese C.S., Martz H.F., "Bayesian Reliability", Springer, 2008.

[20] Wilson A., Fronczyk K., "Bayesian Reliability Combining Information," Institute for Defense Analyses, 2016.

[21] Li M., Meeker W.Q., "Application of Bayesian Methods in Reliability Data Analyses," Journal of Quality Technology, 2014.

[22] Baroth J., Schoefs F., Breysse D., "Construction Reliability, Safety, Variability and Sustainability", John Wiley \& Sons, 2011.

[23] Kelly D., Smith C., "Bayesian Inference for Probabilistic Risk Assessment", Springer, 2011. 


\section{List of Table Captions}

Table 1- Characteristics of the uncertain parameters [N, mm]

Table 2- Common conjugate distributions

\section{List of Figure Captions}

Figure 1- Histogram of the simulated cracks and fitted exponential distribution

Figure 2- Prior and posterior distributions for the uncertain parameter

Figure 3- Dependency of the crack size on the distribution of the rate parameter

Figure 4- Crack size distributions before and after updating

Figure 5- Prior crack size distribution for the numerical method

Figure 6- Likelihood functions for both accurate and inaccurate models

Figure 7- Crack size distributions after several inspections for likelihood function-Case (I)

Figure 8- Crack size distributions after several inspections for likelihood function-Case (II)

Figure 9- Posterior crack size distributions, (a) Conjugate approach, (b) Numerical approach

Figure 10- Prior crack size distributions for multiple locations in conjugate and numerical methods

Figure 11- Crack size distributions for multiple locations in the conjugate method

Figure 12- Crack size distributions for different locations, (a) joint \#1, (b) joint \#2, (c) joint \#8 and (d) joint $\# 10$

Figure 13- Crack size distributions for multiple locations in the numerical method

Figure 14- Posterior crack size distributions for multiple locations in both methods 